\title{
Application of ATR-far-infrared spectroscopy to the analysis of natural resins
}

\author{
Silvia Prati • Giorgia Sciutto • Rocco Mazzeo • \\ Cristian Torri • Daniele Fabbri
}

Received: 28 July 2010 /Revised: 18 October 2010 /Accepted: 25 October 2010 /Published online: 11 November 2010

(C) Springer-Verlag 2010

\begin{abstract}
This study proposes FTIR spectroscopy in the farinfrared region (FarlR) as an alternative method for the characterisation of natural resins. To this purpose, standards of natural resins belonging to four different categories (sesquiterpenic, i.e. elemi, shellac; diterpenic, i.e. colophony, Venice turpentine; diterpenic with polymerised components, i.e. copal, sandarac; triterpenic, i.e. mastic and dammar) used as paint varnishes have been analysed by FarIR spectroscopy in ATR mode. Discrimination between spectral data and repeatability of measurements have been magnified and verified using principal component analysis, in order to verify the effectiveness of the method in distinguishing the four resin categories. The same samples were analysed in the MidIR range, but the spectral differences between the different categories were not evident. Moreover, the method has been tested on historical samples from the painting "La Battaglia di Cialdiran" (sixteenth century) and from a gilded leather (seventeenth century). In the first case, FarIR spectroscopy allowed confirmation of the results obtained by analytical pyrolysis. In the latter, FarIR spectroscopy proved successfully, effective in the identification of the superficial resin layer that could not be detected with the bulk chromatographic analyses.
\end{abstract}

Published in the special issue Analytical Chemistry for Cultural Heritage with Guest Editors Rocco Mazzeo, Silvia Prati, and Aldo Roda.

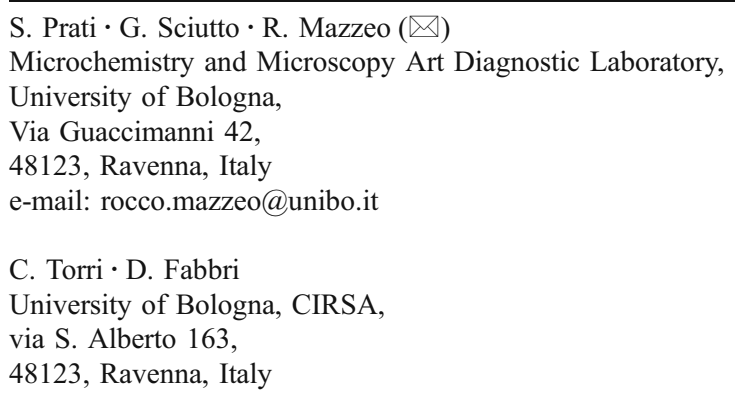

Keywords Cultural heritage · ATR FIR spectroscopy Natural resins $\cdot$ PCA

\section{Introduction}

In the past, tree and insect secretions have been extensively employed in art, as well as in conservation and preservation of art works, thanks to their useful chemical and physical behaviour, including water insolubility, adhesive and coating properties. Different natural resins were mainly used for decorative and varnish purposes since very ancient times for their excellent glasslike capability. A great number of recipes and preparation procedures are documented in literature [1, 2]. Resins mixed with a drying oil were the earliest type of varnish blend, whereas spirit varnishes were introduced only in the sixteenth century. In addition, such biopolymers were mixed with different organic compounds and additives in order to obtain a suitable binding medium [3].

Resins are composed of complex mixtures of terpenoids derived by isoprene. They can be distinguished by their carbon atom numbers into sesqiterpenic (terpenic compounds with 15 carbon atoms), diterpenic (terpenic compounds with 20 carbon atoms) and triterpenic (terpenic compounds with 30 carbon atoms).

Shellac is a sesquiterpenic resin secreted by an insect (Kerria lacca, Keriidae family, Hemiptera order) that infects host trees. The raw lac is scratched from branches and purified through crushing, sieving and water-washing procedures. It is mainly composed of polyesters of oxy acids, which can be dissolved in alcohol, low percentages of waxy substances and low molecular polymers derived from poly hydroxyl acids. Elemi is a malleable material containing a considerable amount of liquid sesquiterpenes and a 
fraction of triterpenic components. The most common type of elemi is obtained from species of the Bursaceae family, growing in the Philippines. Diterpenic resins belong mainly to the Coniferae class (Pinaceae, Cupressaceae, Auracariaceae). Pinaceae resins (i.e. colophony, Venice turpentine, Strasbourg turpentine) are characterised mainly by the presence of abietane and pimarane acids. Cupressaceae (i.e. sandarac) and Auracariaceae (i.e. Manila copal) are composed by labdane acids, in particular by a polymerised fraction derived by communic acids. Triterpenic resins (i.e. dammar and mastic) contain C30 isoprene derivatives showing different basic skeletons, mostly tri- or pentacyclic. Monoric, oleononic, masticadienonic and dammararolic acids are some of the most important triterpenoid components. Mastic can be found on many Mediterranean coasts since it is secreted by the Pistacea lentiscus shrub, while dammar is derived from trees of the Dipterocarpaceae family, growing in the Far East [3].

Several analytical techniques have been so far employed for the identification of resins and the characterisation of their degradation pathway. In particular, GC/MS [4-6] can provide specific information on molecular composition, biological source and degradation effects. However, it is a destructive technique, not allowing the possibility to repeat the analysis and confirm the result.

Py-GC/MS has been widely employed for the characterisation of natural resins [6-10]. This technique is actually less time consuming than GC/MS because no sample pretreatment is required. It also allows to overcome the problem related to the solubility decrease in long time aged resins.

In the last decades, vibrational spectroscopic methods (infrared and Raman) have been optimised to overcome the drawbacks related to bulk analyses, developing portable instruments (for in situ analyses) and microscope-based techniques (for stratigraphical investigations). In particular, infrared spectroscopy has been widely used thanks to its versatility and ability to provide information on both organic and inorganic materials [11]. Since its first introduction, IR spectroscopy has been applied to the examination of paintings and artefacts mainly in the MidIR range 4,000 $600 \mathrm{~cm}^{-1}$ [12]. When micro-sampling accessories for Fourier-transform infrared (FTIR) spectroscopy were introduced in the early 1980s, the following development of FTIR microscopy became an essential tool in the microdestructive analysis of small samples [13]. Moreover, the recent introduction of mapping and imaging equipments allows to collect a large number of FTIR spectra on a surface, producing a distribution map of the identified compounds. FTIR spectroscopy can also be used for nondestructive analyses in form of portable instrument. In recent times, fibre optics have been employed for the development of portable instruments both in the VIS-NearIR and in the MidIR range [14, 15]. Even though FTIR spectroscopy appears to be particularly flexible, only IR-active compounds can be detected. Moreover, the characterisation of the organic substances is a difficult task and only the main class can often be distinguished.

In the field of resinous materials, for instance, FTIR techniques are mainly helpful in recognising their presence, thanks to the presence of both the acid stretching band at around $1,690 \mathrm{~cm}^{-1}$ and the ester triplet due to $\mathrm{C}-\mathrm{O}$ stretching vibrations at around 1,230,1,160, and $1,090 \mathrm{~cm}^{-1}$ (characteristic also of drying oils) [16, 17]. Even though the different resins show some particular features [18], FTIR is mainly applied as a preliminary and non-destructive technique to detect the presence of resinous materials. The subsequent identification of the resins may be accomplished by other techniques, such as GC/MS [19]. FTIR spectroscopy has also been employed to study the ageing effects on natural resins [20, 21], which leads to oxidation cross linking reactions or hydrolysis, depending on the resin typology.

Raman spectroscopy and microscopy have been successively employed for resins characterisation [22-31]. In particular, high wavelengths laser excitation (i.e., 1,064, $780,785 \mathrm{~nm}$ ) has been applied to reduce the fluorescence effects. Despite inherently weaker Raman effect in the nearinfrared region (signal is almost 350 times lower than spectra obtained by using a $250 \mathrm{~nm}$ laser, because of the inverse dependence between molecular scattering intensity and excitation wavelength), the strong reduction of fluorescence effects allows the determination of different resin specimens $[22,26]$. A specific protocol based on the interpretation of the FT-Raman spectra has been developed for the discrimination of diterpenoid and triterpenoid resins [22], considering ageing processes such as oxidation and hydrolysis. The discrimination protocol takes into account the entire spectrum with particular attention to bands from about 3,000 to $500 \mathrm{~cm}^{-1}$. The region from 1,200 to $200 \mathrm{~cm}^{-1}$ is typical of the vibrations of $\mathrm{v}(\mathrm{CC}), \rho(\mathrm{CH})$, $\delta(\mathrm{CCH})$ and $\delta(\mathrm{CCO})$ groups. However, the comparison of resin samples in this region may be difficult because of the inherent weakness of the spectra [24]. Moreover, in some cases, the resins specimen shows fluorescence emissions even in these conditions, which may invalidate their correct classification [22].

In order to avoid a sensible reduction of the Raman signal due to the use of FT lasers, Lau et al. have proposed to employ SERS Raman spectroscopy [31] with the application, on a metal surface ad hoc prepared, of a drop of a solution containing the extracted resin.

This research is aimed at evaluating the use of FTIR spectroscopy in the far-infrared region (FarIR) to propose an alternative and complementary technique for the characterisation of natural resins. FarIR spectroscopy is widely employed in different fields, such as astrophysics, cation exchange and interactions in ceramics, as well as lattice vibrations in the field of polymer crystallisation. 
Since 1969, several studies have highlighted the analytical potential of FarIR spectroscopy for the investigation of minerals in the $200-50 \mathrm{~cm}^{-1}$ region [32]. Furthermore, recent studies document the application of FarIR spectroscopy in both transmission [33] and attenuated total reflectance (ATR) modes [34-37] as a valid alternative method for the characterization of the compounds inactive in the MidIR (different inorganic pigments and corrosion products). As far as the authors know, the application of FTIR spectroscopy in the FarIR region for the investigation

Table 1 Natural resins signals in the FarIR region obtained in ATR

\begin{tabular}{|c|c|c|c|c|c|c|c|c|}
\hline Elemi & Shellac & Colophony & Venice turpentine & Sandarac & Copal & Dammar & Mastic & Approx assignement \\
\hline & & & & $696(\mathrm{~m})$ & $695(\mathrm{~m})$ & & $692(w)$ & \\
\hline & & & & $685(\mathrm{~m})$ & & & & \\
\hline & & & & & $675(\mathrm{~m})$ & & & \\
\hline & & $658(\mathrm{~s})$ & $652(\mathrm{~s})$ & & & $658(\mathrm{~m})$ & & \\
\hline \multirow[t]{2}{*}{$663(\mathrm{~s})$} & & & & & & & & $v \mathrm{CC}^{25}$ \\
\hline & $646(\mathrm{~m})$ & & & & & & $646(\mathrm{~m})$ & $\delta \mathrm{CCC}^{25}$ \\
\hline \multirow[t]{3}{*}{$638(w, s h)$} & & & & & & $638(\mathrm{~m})$ & & $\delta \mathrm{CCC}^{25}$ \\
\hline & & $611(w)$ & $610(w, s h)$ & & $617(\mathrm{~m})$ & & & \\
\hline & & & & & & $601(\mathrm{w})$ & & \\
\hline \multicolumn{9}{|l|}{$590(\mathrm{~m})$} \\
\hline & & & & $581(\mathrm{~m})$ & $583(\mathrm{~m})$ & $580(\mathrm{~s})$ & $583(\mathrm{~s})$ & \\
\hline & $572(\mathrm{~m})$ & & & & & & & \\
\hline \multirow[t]{2}{*}{$562(\mathrm{~m})$} & & $561(\mathrm{~m}, \mathrm{sh})$ & $560(\mathrm{~m}, \mathrm{sh})$ & $556(\mathrm{~m})$ & $563(\mathrm{~m})$ & & & $\delta \mathrm{CCO}^{31}$ \\
\hline & & $540(\mathrm{~s})$ & $538(\mathrm{~m})$ & & & & & $\delta \mathrm{CCO}^{25}$ \\
\hline \multirow[t]{3}{*}{$528(\mathrm{~m})$} & & & & & & $530(\mathrm{~s})$ & $526(\mathrm{~m})$ & $\delta \mathrm{CCO}, \delta \mathrm{CCC}^{24}$ \\
\hline & & & & $520(\mathrm{~m})$ & $520(\mathrm{~m})$ & & & \\
\hline & $518(\mathrm{~s})$ & & & & & & & \\
\hline \multirow[t]{2}{*}{498 (m, sh) } & & & & & $491(w)$ & & & \\
\hline & & $478(w)$ & $475(w)$ & & & & $471(\mathrm{~m})$ & $\delta \mathrm{CCC}^{27}$ \\
\hline \multirow[t]{2}{*}{$460(\mathrm{~m})$} & $460(\mathrm{~m})$ & & & & & $458(\mathrm{~m})$ & & $\delta \mathrm{CO}^{31}$ \\
\hline & & $449(w)$ & & $449(\mathrm{~m})$ & 447 (m) & & $451(w)$ & $\delta \mathrm{CCO}^{26,27}$ \\
\hline \multirow[t]{5}{*}{$433(\mathrm{~m})$} & & & & & & & $431(w)$ & \\
\hline & & $417(\mathrm{~m})$ & $422(w)$ & & & & & \\
\hline & $411(\mathrm{~m})$ & & & & & & & \\
\hline & & & & $397(\mathrm{~s})$ & $395(\mathrm{~s})$ & & & \\
\hline & & $393(\mathrm{~m})$ & $387(\mathrm{~m})$ & & & & & \\
\hline \multirow[t]{3}{*}{$367(\mathrm{~m})$} & & & & $363(\mathrm{~m}, \mathrm{sh})$ & $362(\mathrm{~m}, \mathrm{sh})$ & & & $\delta \mathrm{CCC}^{31}$ \\
\hline & $356(w)$ & & & & & $351(\mathrm{~m})$ & & \\
\hline & & $345(\mathrm{~m})$ & $347(w)$ & & & & & \\
\hline \multirow[t]{2}{*}{$340(\mathrm{~m})$} & $341(w)$ & & & & & & $338(\mathrm{~s})$ & \\
\hline & $313(w)$ & & & $310(w)$ & & $318(w)$ & & $\tau(\mathrm{CCC}), \tau(\mathrm{CCO})^{2}$ \\
\hline \multirow[t]{2}{*}{$294(\mathrm{~m})$} & $195(w)$ & $290(\mathrm{~m})$ & $286(\mathrm{~m})$ & & & & & \\
\hline & & & & & & & $269(w)$ & \\
\hline \multirow[t]{3}{*}{$265(w)$} & $265(\mathrm{~m})$ & & & $260(\mathrm{~m})$ & $265(\mathrm{~m})$ & & & $\tau\left(\mathrm{CH}_{3}\right)^{26,27}$ \\
\hline & & $253(w)$ & $252(\mathrm{~m})$ & & & & & \\
\hline & & & & & & $243(\mathrm{~m})$ & & \\
\hline \multicolumn{9}{|l|}{$229(\mathrm{~m})$} \\
\hline & $212(\mathrm{~m})$ & & & & $212(\mathrm{~m})$ & & & \\
\hline & & & & 199 (m) & & & $199(w)$ & \\
\hline \multicolumn{9}{|l|}{$189(\mathrm{~m})$} \\
\hline & & & & & $169(\mathrm{w})$ & $169(\mathrm{~m})$ & $170(\mathrm{~m})$ & \\
\hline
\end{tabular}

$s$ strong, $m$ medium, $w$ weak, $s h$ shoulder 


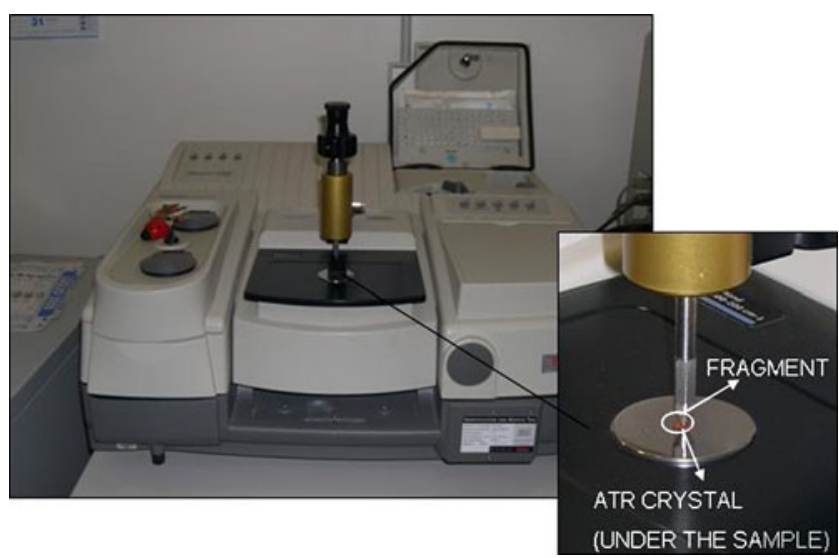

Fig. 1 ATR Smart Orbit ${ }^{\mathrm{TM}}$ mounted on a Thermo Nicolet Nexus 5700 spectrometer

of organic substances has not yet been carried out. To this purpose, standards of natural resins used as paint varnishes have been analysed by FarIR spectroscopy in ATR mode, obtaining promising results. Moreover, the discrimination between spectral data was magnified and verified using principal component analysis (PCA).

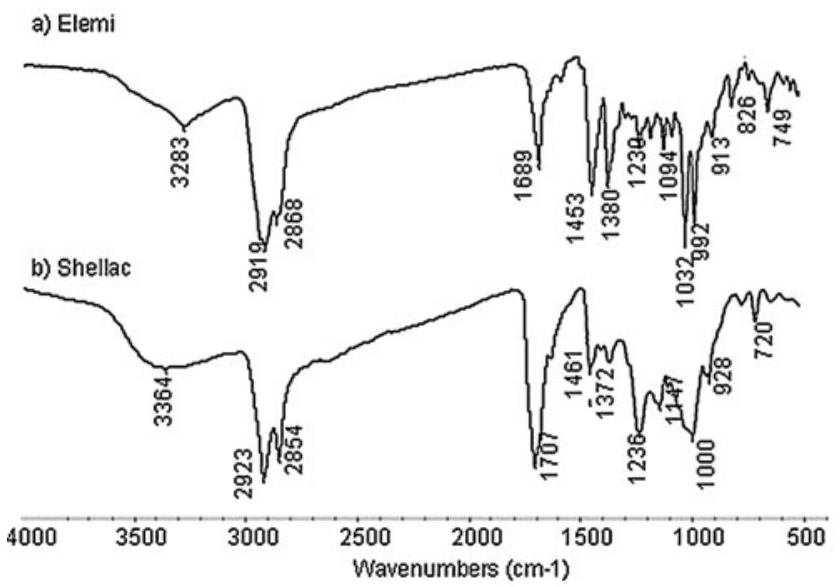

c) Colophony
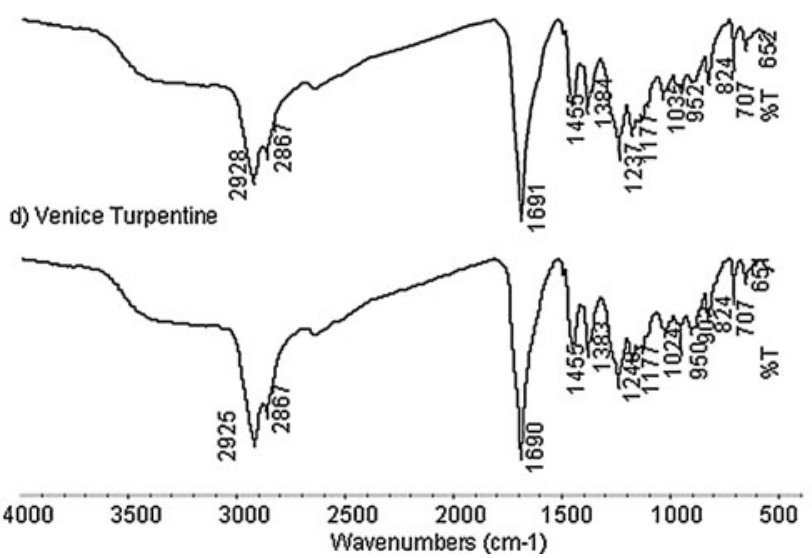

The ability of the method for identification purposes in real matrices was tested by the analysis of historical samples. The results were compared to those obtained from a complementary methodology based on analytical pyrolysis.

\section{Experimental}

Materials

All the standard materials (see Table 1) were purchased from Kremer. Solid resins were analysed as samples in a powder form, collected from the bulk specimen. Venice turpentine (which is in the liquid form) was analysed only after preparing a resin film obtained by its solubilisation in ethyl alcohol and application on a glass slide support.

Two historical samples have been examined. One (BP) was collected from a yellow area of the "La Battaglia di Cialdiran", an oil painting on canvas dated back to the sixteenth century, the work of an unknown artist conserved in the Regional Museum of Mirto Palace, Palermo, Italy.
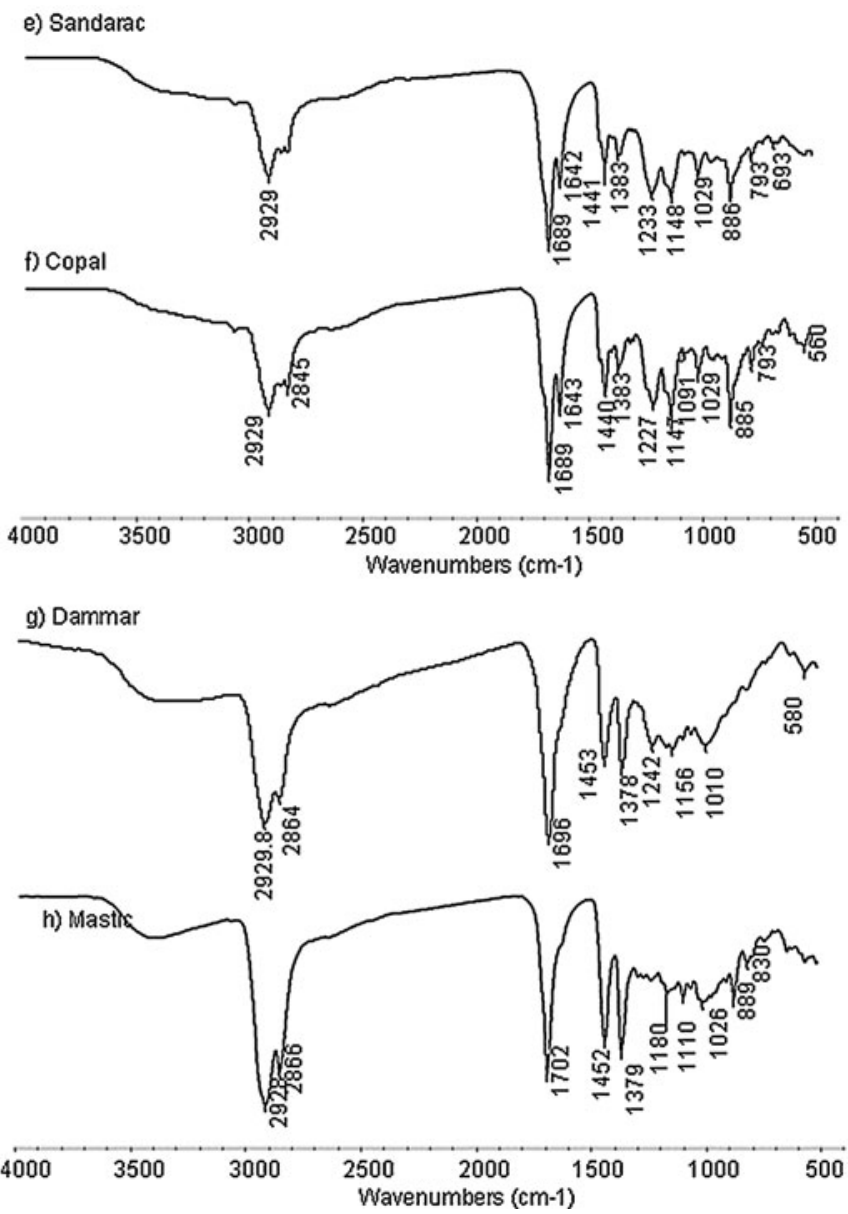

Fig. 2 MidIR spectra obtained in ATR of a elemi, $\mathbf{b}$ shellac, $\mathbf{c}$ colophony, $\mathbf{d}$ Venice turpentine, e sandarac, $\mathbf{f}$ copal, $\mathbf{g}$ dammar, $\mathbf{h}$ mastic 
The second (PMB7) was a gilded leather sampled from a painted wall hanging dated to the seventeenth century.

\section{FarIR spectroscopy}

A Thermo Nicolet Nexus 5,700 spectrometer, using a solid-substrate beamsplitter operating in the FarIR region of $700-90 \mathrm{~cm}^{-1}$, was employed. A Parker/Balston selfcontained purge gas generator was used to minimise interference bands generated from ambient moisture. During the collection of spectra the outlet pressure was set at $30 \mathrm{psi} / \mathrm{bar}$, while the outlet flow control at 9 (dimensionless).

The ATR spectra were registered by a diamond ATR Smart Orbit ${ }^{\mathrm{TM}}$ accessory (from Thermo Optec) both in the MidIR (DTGS detector with a $\mathrm{KBr}$ window) and in the FarIR (DTGS detector with a polyethylene window) region (Fig. 1). The diamond crystal has a refractive index of 2.4, with a single bouncing refractive infrared beam at $45^{\circ}$ angle of incidence. Analyses were performed on samples in a powder form by grinding the bulk resins, or in small fragments after sampling thin films from resin. Measurements were carried out at a spectral resolution of $4 \mathrm{~cm}^{-1}$, with a physical mirror rate of $0.6329 \mathrm{~cm} \mathrm{~s}^{-1}$. Approximately, less than $0.5 \mathrm{mg}$ of material was required for each measurement. Several replicate spectra were collected for each resin specimen.

The historical samples were analysed placing the external surface of the fragments on the macro ATR crystal, simply applying a pressure to optimise the contact.

\section{Chemometric analysis}

Spectral data were processed by PCA and employing the chemometric package V-PARVUS. Matrix dataset was composed with intensity values of peaks as variables (columns) and spectra in the range $670-150 \mathrm{~cm}^{-1}$ as objects (rows). Standard normal variate (SNV) transformation and column centering were applied as a row pre-treatment on the matrix dataset. SNV was specially applied in order to remove slope variation and to correct both baseline shifts and global intensity variations [38].
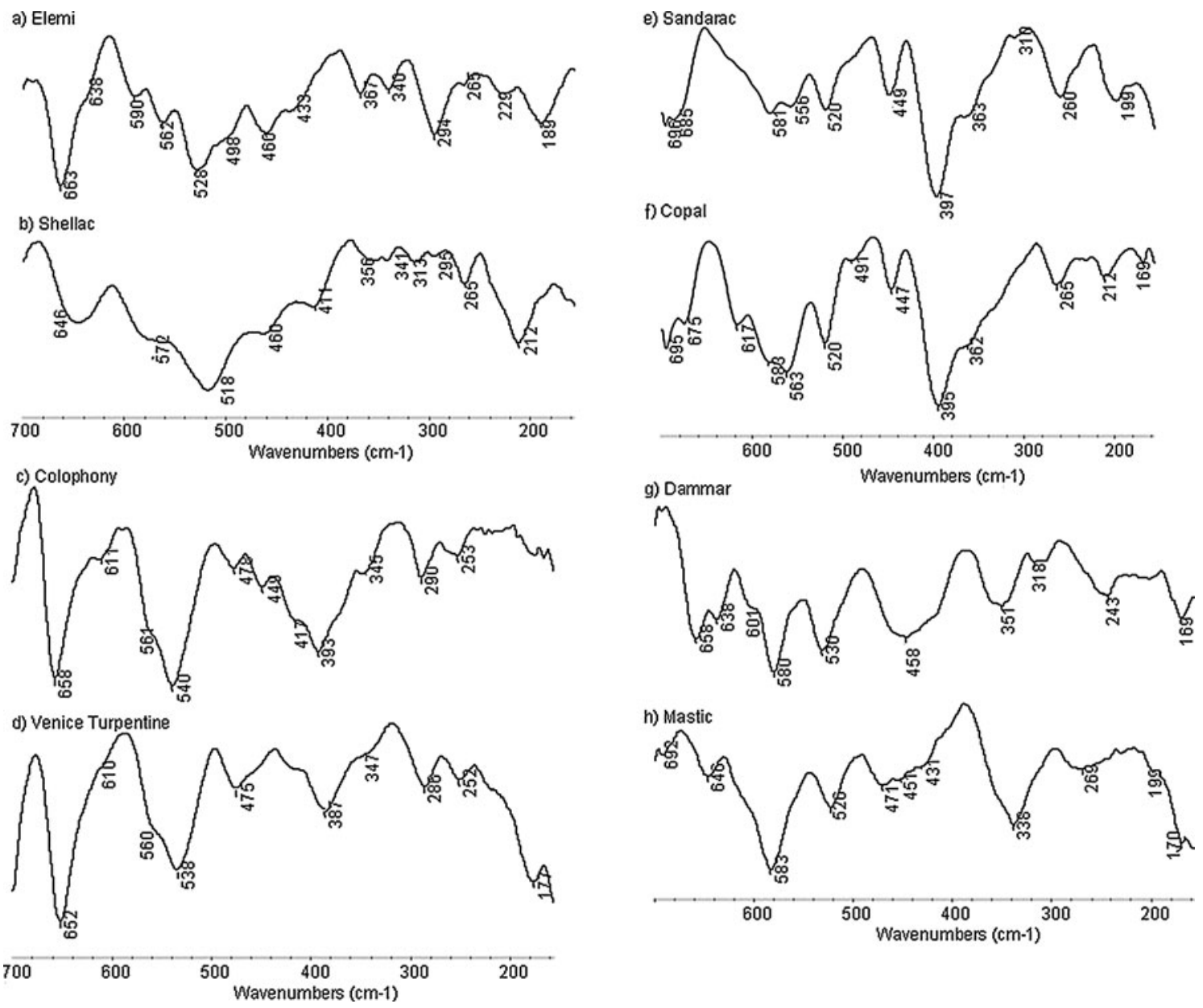

Fig. 3 FarIR spectra obtained in ATR of a elemi, $\mathbf{b}$ shellac, $\mathbf{c}$ colophony, $\mathbf{d}$ Venice turpentine, e sandarac, $\mathbf{f}$ copal, $\mathbf{g}$ dammar, $\mathbf{h}$ mastic 
Analytical pyrolysis

The historical samples BP and PBM7 were analysed by offline pyrolysis with a CDS 1,000 Pyroprobe (Platinum filament coil) following the procedure proposed by Torri and Fabbri [39]. Shortly, samples were pyrolysed at $700{ }^{\circ} \mathrm{C}$ for $60 \mathrm{~s}$, evolved pyrolysis products were sampled by solidphase microextraction with a carboxen-PDMS fibre. The fibre was inserted into the injector port kept at $280^{\circ} \mathrm{C}$ of a 6850 Agilent HP gas chromatograph connected to a 5975 Agilent HP quadrupole mass spectrometer. Analytes were separated by a HP-5MS fused-silica capillary column (stationary phase poly $[5 \%$ diphenyl $/ 95 \%$ dimethyl]siloxane, $30 \mathrm{~m}, 0.25 \mathrm{~mm}$ i.d., $0.25 \mu \mathrm{m}$ film thickness) using helium as carrier gas. Mass spectra were recorded under electron ionisation $(70 \mathrm{eV})$ at a frequency of 1 scan s $\mathrm{s}^{-1}$ within the $12-450 \mathrm{~m} / \mathrm{z}$ range. The following thermal programme was used: $35{ }^{\circ} \mathrm{C}$ for $5 \mathrm{~min}$, then $10{ }^{\circ} \mathrm{C} / \mathrm{min}$ until $310^{\circ} \mathrm{C}$ followed by a column cleaning at $310^{\circ} \mathrm{C}$ for $3 \mathrm{~min}$. GC/MS traces of real samples were compared to those derived from analytical pyrolysis of standard resins.

\section{Results and discussions}

The use of a macro diamond accessory had three main advantages: no sample manipulation was required; the amount of sample was reduced, according with conservation purposes; the varnish applied as external layer on historical samples could be directly analysed just positioning the external sample surface onto the ATR crystal.

\section{Standard resins}

The different resin specimens have been analysed both in the MidIR and in the FarIR region. The ATR-MidIR spectra (Fig. 2) showed extremely similar profiles. In fact it is difficult to identify not only the different resins but also more generic categories such as: (a) sesquiterpenic resins, (b) diterpenic resins containing abietane compounds, (c) diterpenic resins containing polymerised communic acid, (d) triterpenic resins.

On the other hand, FarIR analyses allowed the identification of specific bands for each investigated category as shown in Table 1.

Elemi and shellac show characteristic FarIR spectra (Fig. 3a-b) allowing a clear distinction between them. Some similarities, in terms of frequency values, can be observed comparing the elemi spectrum with the mastic and dammar ones, even though the resins specimens can be easily distinguished (Table 1).

Diterpenic resins have a common peak at around $395 \mathrm{~cm}^{-1}$ but they also show particular features allowing to distinguish Pinacea resins, such as colophony and Venice turpentine (Fig. 3c-d), from diterpenic resins containing polymerised communic acid, such as sandarac and copal (Fig. 3e-f). Colophony and Venice turpentine are characterised by the presence of peaks at around 540 and $475 \mathrm{~cm}^{-1}$, while sandarac and copal have peaks at 520 and $260 \mathrm{~cm}^{-1}$ (Fig. 3c-f).

Triterpenic resins show significant similarities in all the collected spectra having common features, such as the peak at about $580 \mathrm{~cm}^{-1}$. Some differences can be noticed between the two triterpenic resins which can be used to discriminate them, such as the band at 658, 638, and $351 \mathrm{~cm}^{-1}$ typical of dammar and bands at 646 and $338 \mathrm{~cm}^{-1}$ mainly present in mastic resin (Fig. $3 \mathrm{~g}-\mathrm{h}$ ).

Previous studies on the characterization of natural resins performed by Raman spectroscopy allowed the approximate assignment of frequency values in the vibrational mode [22-31]. A comparison with the data reported in literature was made to identify possible transitions, which are active both in infrared and Raman spectroscopy. FarIR spectra are indeed different from Raman ones, but there are common absorptions. In particular the following vibrations $v$ $\mathrm{CC}^{25}, \delta \mathrm{CCC}^{24-25,27}, \delta \mathrm{CCO}^{23-27,31}, \delta \mathrm{CCC}^{24,31}, \delta \mathrm{CO}^{31}$, $\tau(\mathrm{CCC})^{24}, \tau(\mathrm{CCO})^{24}$ and $\tau\left(\mathrm{CH}_{3}\right)^{26,}{ }^{27}$ have been tentatively assigned in Table 1 . The presence of vibrational modes active only in the FarIR region further confirms that the two techniques may complement each other in the characterisation of natural resins.

\section{a)}

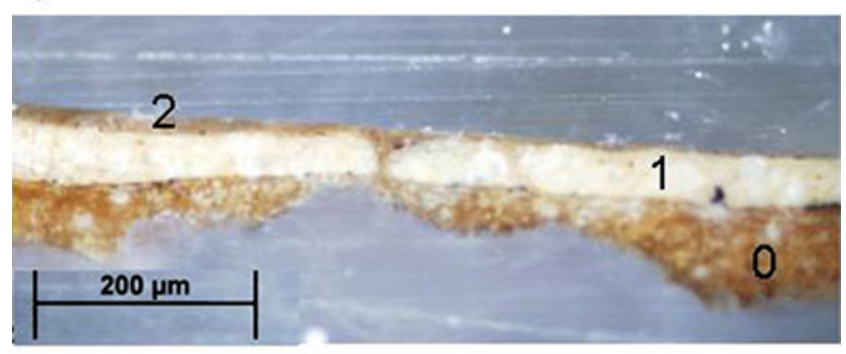

\section{b)}

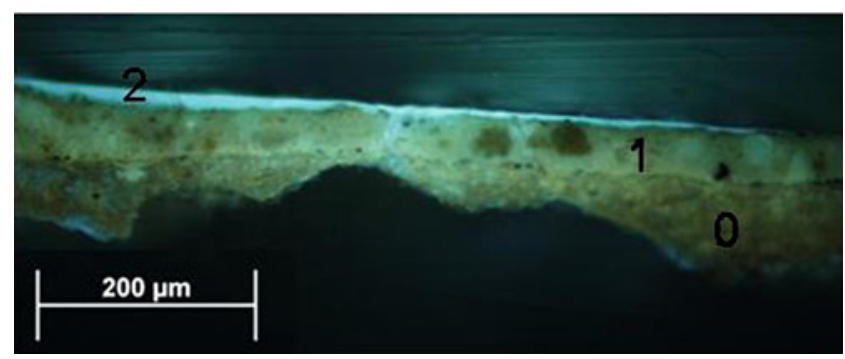

Fig. 4 Cross-section photomicrographs under a visible and b UV light of the $\mathrm{KBr}$ embedded $\mathrm{BP}$ cross-section (layer 2 varnish; layer 1 pigmented layer composed by yellow ochre, lead white, calcite, siccative oil; layer 0 preparation composed by gypsum) 


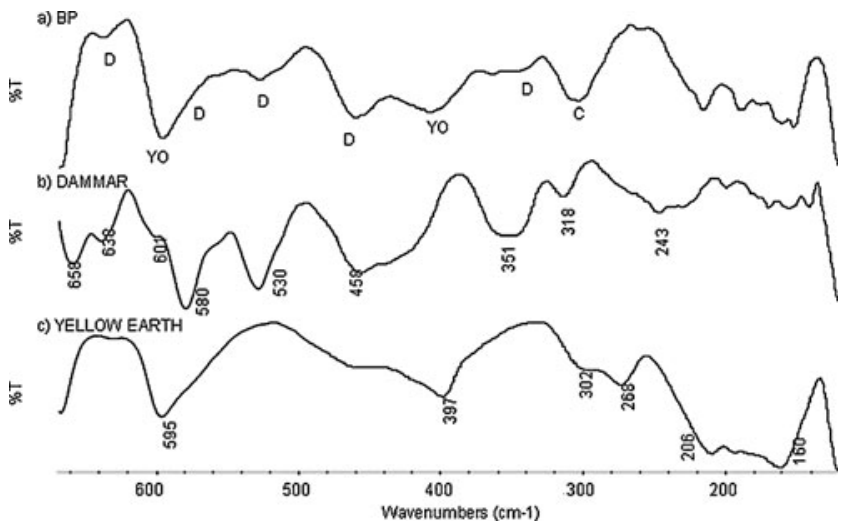

Fig. 5 FarIR spectra obtained in ATR of a BP sample, b dammar $(D)$ c yellow ochre $(Y O)$ : $C$ calcium carbonate

Historical samples

The cross-section photomicrographs of the BP sample shown in Fig. 4 allows to clearly identify the presence of an organic protective applied as external layer. This may be revealed by the strong whitish fluorescence visible under UV light, which is not influenced by the pollution of the embedding material because the sample was embedded in $\mathrm{KBr}[40]$.

Figure 5a shows the BP spectrum in the FarIR region obtained in macro ATR on the external surface (corresponding to layer 2 in Fig. 4). From the MidIR spectrum (not reported) the carboxylic acids band $\left(1,716 \mathrm{~cm}^{-1}\right)$ suggests the presence of a natural resin, but its identification resulted rather difficult. The FarIR spectrum was more informative and when comparing it with the dammar profile (Fig. 5b) different analogies can be noticed in the position and shape of the bands (i.e. bands at 638, $580,530,458,351 \mathrm{~cm}^{-1}$ ). The occurrence of dammar in this sample suggested by FarIR data was supported by analytical pyrolysis. Chromatograms in full scan obtained from off-line pyrolysis of sample BP and dammar are shown in Fig. 6a and b, respectively. A cluster of peaks with retention times and mass spectra corresponding to those of dehydrogenated sesquiterpenes $\mathrm{C}_{15} \mathrm{H}_{22}$ specific of
Fig. $6 \mathrm{GC} / \mathrm{MS}$ of pyrolysate from a sample BP and $\mathbf{b}$ dammar resin; mass chromatograms at $\mathrm{m} / \mathrm{z} 159$ expanded in elution region of dammar markers $\left(\mathrm{C}_{15} \mathrm{H}_{22}\right.$ bicyclic sesquiterpenoids) of $\mathbf{c}$ sample BP and $\mathbf{d}$ dammar resin, e mass spectrum of the GC peak at $19.3 \mathrm{~min}$ tentatively identified as calamenene

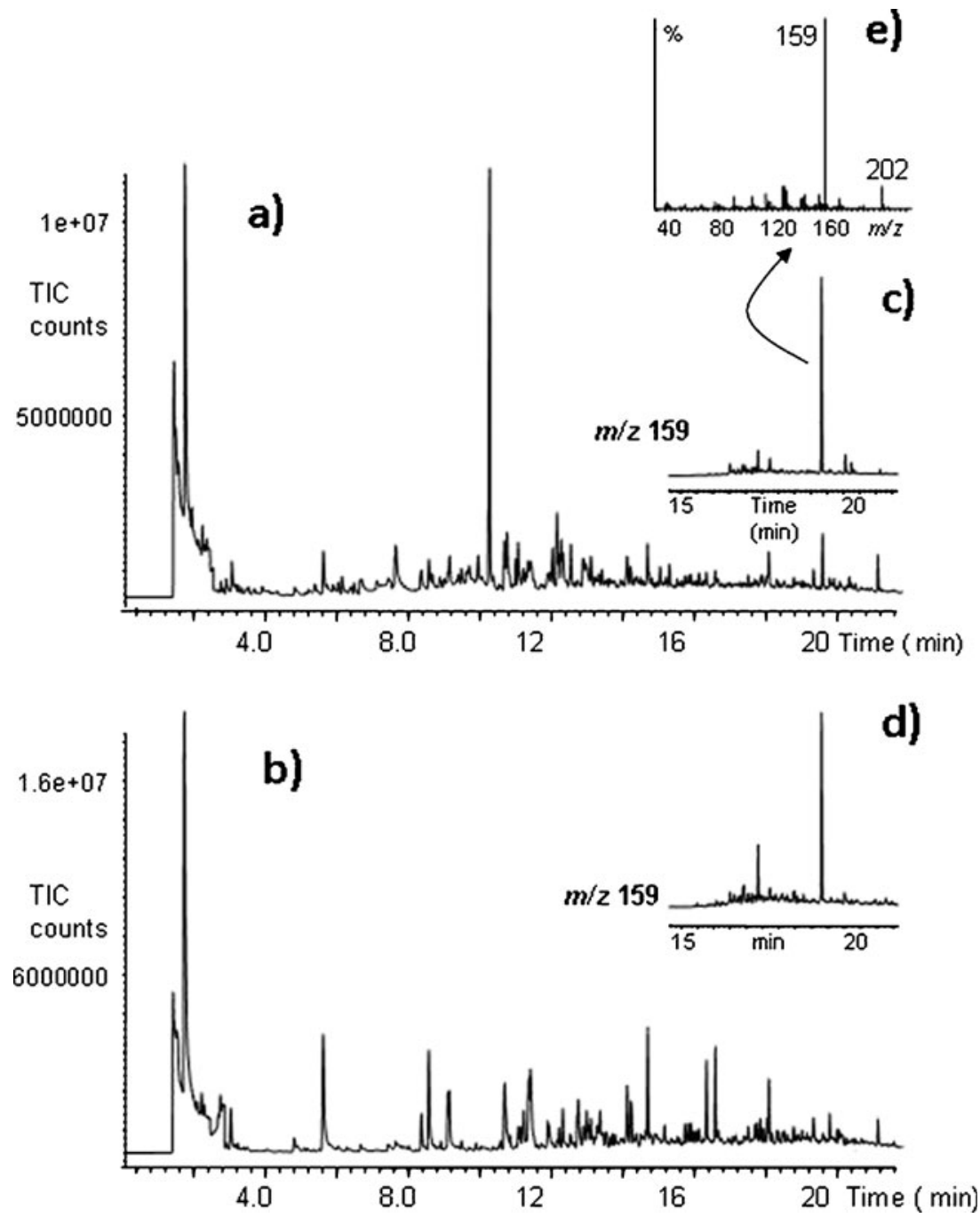


dammar [8] were revealed in both samples. In particular, the mass chromatograms at $\mathrm{m} / \mathrm{z} 159$ in Fig. 6c and d show a peak at $19.3 \mathrm{~min}$ tentatively identified from its mass spectrum (Fig. 6e) as calamenene (4-isopropyl-1,6-dimethyl-1,2,3,4-tetrahydro-naphthalene), a distinctive marker of dammar resin [41].

The interpretation of FarIR spectrum was complicated by signals arising from the matrix, in particular, silicatebased pigments (Fig. 5c), such as a yellow earth (595 and $\left.397 \mathrm{~cm}^{-1}\right)$, and calcium carbonate $\left(307 \mathrm{~cm}^{-1}\right)$ [36]. These compounds derived from the underlying yellow layer (layer 1 in Fig. 4) after the sample squeezing due to the contact with the ATR crystal, as it was confirmed by micro FTIR in ATR in the MidIR range directly on the stratigraphy.

This represents a limitation of the method because in order to obtain meaningful spectra, it is necessary to apply pressure which might partially disaggregate the sample. Moreover, strong absorbent inorganic salts may overlap with the signal arising from the resins. In this case, the resin layer, as shown in the stratigraphy (Fig. 4b) is homogeneously present and, although very thin, its spectrum can still be detected. To overcome this problem, a new system is being developed, aimed at analysing small particles, sampled under the stereomicroscope from the external layer, in transmission mode using an IR condenser.

The cross-section of sample PMB7 is reported in Fig. 7. Gilded leathers in the past were usually made by glueing a very thin leaf of tin or silver over the support which was afterwards varnished with an oleo-resin binder (mainly shellac, sandarac or colophony) mixed with yellow dyes [2].

Micro FTIR analyses in the MidIR range have been performed in reflection-absorption spectroscopy. The varnish layer is in fact $20 \mu \mathrm{m}$ thick and the metallic leaf allows to reflect back the infrared light so that the final spectrum is similar to that obtained in transmission.

As for sample BP, the presence of resinous materials is suggested by the carboxylic acids band $\left(1,704 \mathrm{~cm}^{-1}\right)$ but it is not possible to assign the spectrum to any particular resin. On the other hand, the FarIR spectrum (Fig. 8) shows the absorption at 540 and $393 \mathrm{~cm}^{-1}$ suggesting the presence of a diterpenic resin without the polymerised component (i.e. colophony or Venice turpentine). In particular, colophony appears among the ingredients mentioned by old recipes [2]. Peaks 529 and $340 \mathrm{~cm}^{-1}$ may derive from the underlying leather support (Fig. 8c) while the other unknown peaks might be connected to the dye used to provide the gilded coloration. Further investigations aimed at characterising the possible presence of organic dyes (i.e. saffron, aloe, antraquinonic dyes) are still being carried out by FarIR/MidIR spectroscopy as well as other techniques such as Raman spectroscopy and HPLC-DAD.

About $0.5 \mathrm{mg}$ of the organic layer was scraped off from the same fragment. The sampling was performed under the UV

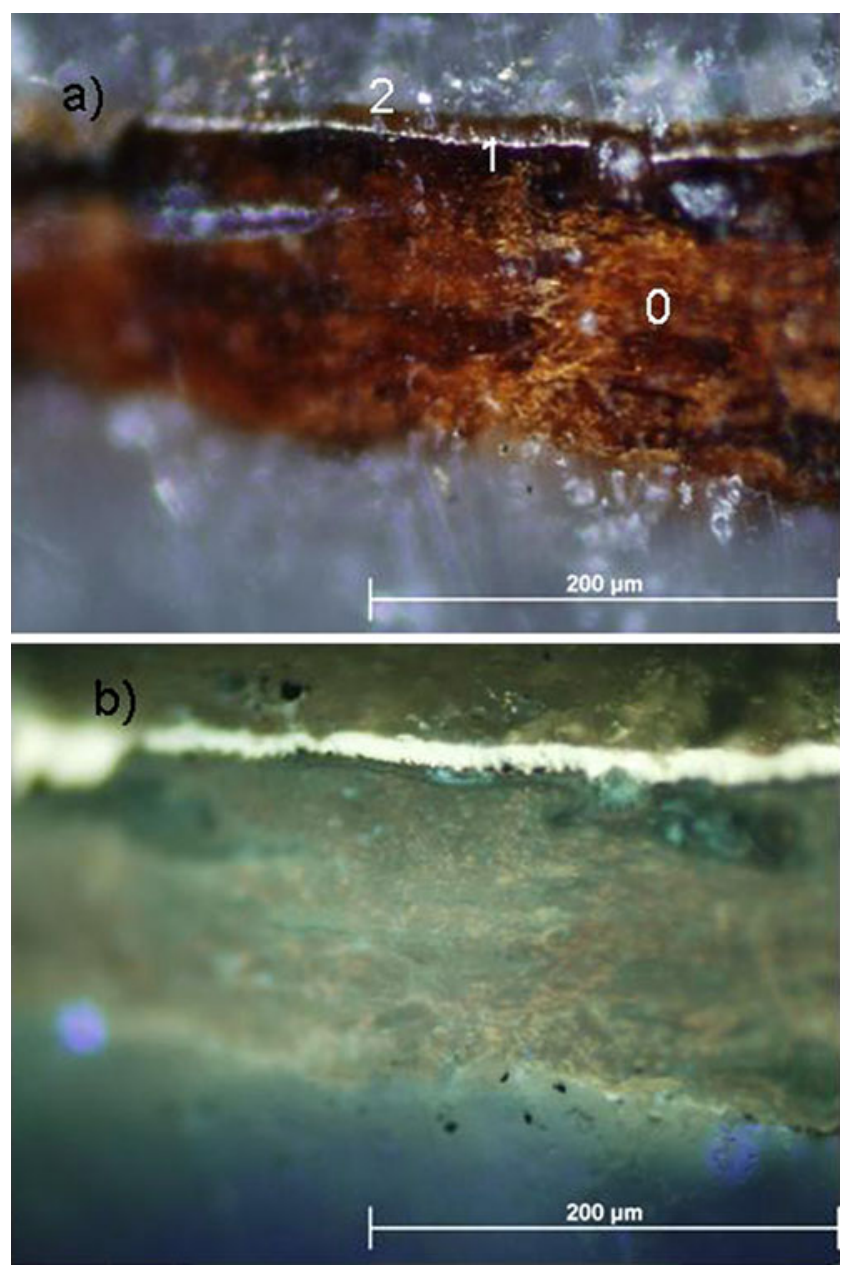

Fig. 7 Cross-section photomicrographs under a visible and b UV light of the $\mathrm{KBr}$ embedded PMB7 cross-section (layer 2 gold varnish, layer 1 Silver leaf, layer 0 leather)

light illumination of an optical microscope in order to better localise the higher fluorescence generated by the varnish layer. However, analytical pyrolysis of sample PBM7 failed to confirm the occurrence of resins since the MS-pyrograms were dominated by intense peaks associated to the thermal degradation products of proteins derived from the leather support.

\section{Data analysis}

A statistical multivariate analysis was performed in order to evaluate the dispersion of the data and to better characterise the different resins, since PCA is usually employed for data exploration purposes, outliers detection, visualisation of graphical clustering and evaluation of the information present in the spectral signals [42].

PCA was performed both on MidIR and on FarIR data and the results were compared. The PC1/PC2 score plot obtained with MidIR spectra is reported on Fig. 9. These two PCs are able to clearly discriminate only some resins 


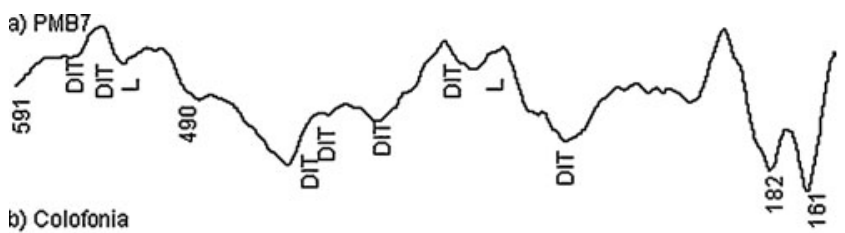

b) Colofonia
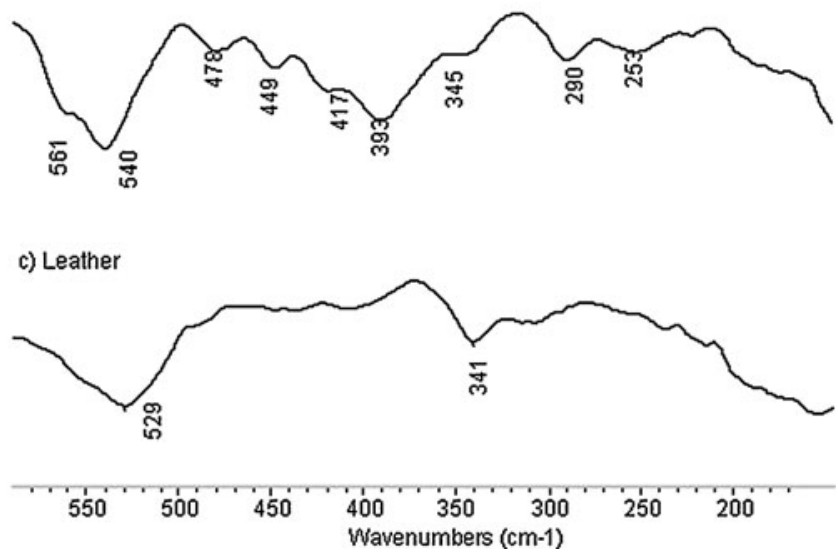

Fig. 8 FarIR spectra obtained in ATR of a PMB7 sample, b colophony c leather (DIT diterpenic resin without a polymerised component; $L$ leather)

such as dammar, mastic, shellac and elemi, while are not effective for the diterpenic resins with or without the polymerised fraction. Moreover, the resins dispersion between the replicated spectra is in some cases comparable with the dispersion between different standards (i.e. mastic and Venice turpentine dispersions). Furthermore, PCA is not able to correctly identify which resin is present in the historical sample BP. Indeed, the two available replicates are positioned in a region far from all the analysed resins. This may suggest that the MidIR region is more influenced

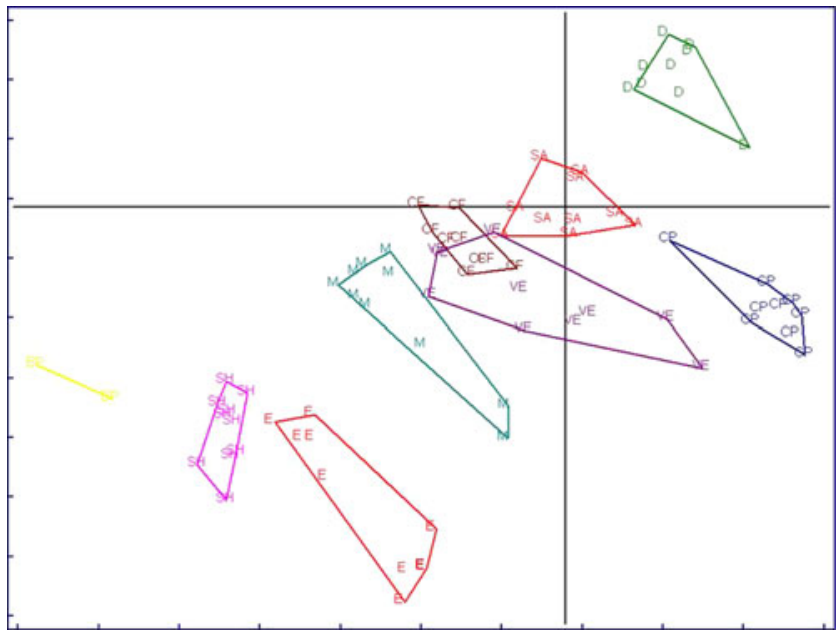

Fig. 9 PC1/PC2 plot resulting from PCA of the resins MidIR spectra (minimum nine replicates for standards, two replicates for the BP sample); $B P$ historical sample, $C F$ colophony, $C P$ copal, $D$ dammar, $E$ elemi, $M$ mastic, $S A$ sandarac, $S H$ shellac, $V E$ Venice turpentine by the presence of other specimens present in the historical sample, which affect the PCA results.

PC1/PC2 score plot obtained with FarIR spectra is reported in Fig. 10. These two PCs are able to wellidentify four clustered groups, thanks to the lower variability of replicated spectra compared with the dispersion between the different standards. It can be noted that the four described categories are clearly separated. The statistical multivariate approach allowed to distinguish resins belonging to the same categories (i.e. mastic from dammar, shellac from elemi). That was not true when considering diterpenic resins and diterpenic with the polymerised fraction: although copal and sandarac appeared separated, the spectral profiles resulted extremely similar and the differences might become even less evident when analysing historical/aged samples.

PCA confirmed the interpretation also in the case of the historical sample BP. Even though the FarIR spectra contain other compounds, the four replicates are positioned in the dammar region. One replicate is a bit shifted, probably because of a greater influence by the other compounds arising for the underlying layer. It was not possible to apply PCA on sample PMB7 because the small fragment available allowed just one FarIR and Py-GC/MS analysis.

The Fisher weights (FW) [43, 44] were calculated in order to provide an estimation of the discriminative ability of each PC in terms of separation between two categories (Tables 2 and 3).

For MidIR data, the FW (Table 2) were generally lower than those obtained with the FarIR spectra (Table 3). This suggests that the resins are less discriminated. It is interesting to notice the high FW values calculated between the BP classes and all the resin classes, suggesting that, in

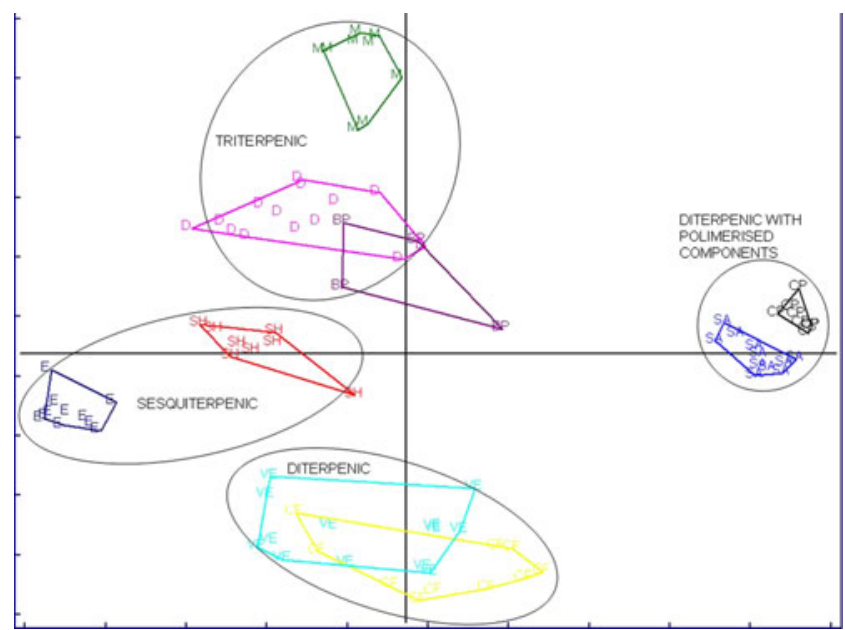

Fig. $10 \mathrm{PC} 1 / \mathrm{PC} 2$ plot resulting from PCA of the resins FarIR spectra (minimum eight replicates for standards, four replicates for the BP sample); $B P$ historical sample, $C F$ colophony, $C P$ copal, $D$ dammar, $E$ elemi, $M$ mastic, $S A$ sandarac, $S H$ shellac, $V E$ Venice turpentine 
Table 2 Fisher weights calculated as between classes variance to within class variance ratio for MidIR spectra; $B P$ historical sample; $C F$ colophony; $C P$ copal, $D$ dammar; $E$ elemi; $M$ mastic; $S A$ sandarac; $S H$ shellac; $V E$ Venice turpentine

\begin{tabular}{|c|c|c|}
\hline & PC1 FW & PC2 FW \\
\hline CP-E & 33.0 & 6.8 \\
\hline CP-M & 27.4 & 0.0 \\
\hline $\mathrm{CP}-\mathrm{CF}$ & 40.2 & 2.8 \\
\hline CP-D & 3.2 & 28.0 \\
\hline CP-SH & 172.9 & 6.9 \\
\hline CP-VE & 5.5 & 0.0 \\
\hline CP-SA & 13.2 & 6.3 \\
\hline CP-BP & 164.3 & 4.5 \\
\hline E-M & 0.2 & 4.3 \\
\hline $\mathrm{E}-\mathrm{CF}$ & 2.3 & 12.8 \\
\hline E-D & 22.0 & 32.0 \\
\hline E-SH & 5.3 & 0.9 \\
\hline E-VE & 2.6 & 6.7 \\
\hline E-SA & 8.2 & 16.3 \\
\hline E-BP & 17.4 & 2.9 \\
\hline $\mathrm{M}-\mathrm{CF}$ & 0.9 & 1.2 \\
\hline M-D & 17.2 & 12.4 \\
\hline M-SH & 8.7 & 2.8 \\
\hline $\mathrm{M}-\mathrm{VE}$ & 1.6 & 0.0 \\
\hline M-SA & 5.5 & 2.7 \\
\hline M-BP & 22.6 & 1.2 \\
\hline CF-D & 25.0 & 17.8 \\
\hline CF-SH & 60.4 & 19.2 \\
\hline CF-VE & 0.6 & 1.4 \\
\hline CF-SA & 4.5 & 1.0 \\
\hline CF-BP & 69.9 & 22.1 \\
\hline D-SH & 167.3 & 55.9 \\
\hline D-VE & 2.1 & 19.0 \\
\hline D-SA & 4.8 & 10.2 \\
\hline D-BP & 148.5 & 72.2 \\
\hline SH -VE & 11.6 & 6.2 \\
\hline SH-SA & 67.2 & 25.9 \\
\hline SH-BP & 15.0 & 1.5 \\
\hline VE-SA & 0.1 & 3.6 \\
\hline VE-BP & 22.5 & 3.7 \\
\hline SA-BP & 81.9 & 31.4 \\
\hline
\end{tabular}

this case, PCA is unable to identify the historical sample composition.

Fisher Weights referred to the FirIR spectra highlighted the discriminative ability of $\mathrm{PC} 1$ and $\mathrm{PC} 2$ in the distinction of the resin specimens under investigation, according to their chemical nature and to the information achieved from the observation of the spectra. In particular, the higher values of $\mathrm{FW}$ were related to the separation between
Table 3 Fisher weights calculated as between classes variance to within class variance ratio for FarIR spectra; $B P$ historical sample; $C F$ colophony; $C P$ copal, $D$ dammar; $E$ elemi; $M$ mastic; $S A$ sandarac; $S H$ shellac; $V E$ Venice turpentine

\begin{tabular}{|c|c|c|}
\hline & PC1 FW & PC2 FW \\
\hline CP-E & 812.8 & 21.5 \\
\hline CP-M & 316.7 & 45.2 \\
\hline $\mathrm{CP}-\mathrm{CF}$ & 18.7 & 75.0 \\
\hline CP-D & 55.9 & 14.4 \\
\hline CP-SH & 138.4 & 1.5 \\
\hline CP-VE & 28.9 & 44.3 \\
\hline CP-SA & 2.5 & 3.3 \\
\hline CP-BP & 35.5 & 1.2 \\
\hline E-M & 81.2 & 81.55 \\
\hline $\mathrm{E}-\mathrm{CF}$ & 18.9 & 26.4 \\
\hline E-D & 10.9 & 50.24 \\
\hline E-SH & 12.9 & 6.22 \\
\hline E-VE & 11.6 & 12.9 \\
\hline E-SA & 420 & 6.3 \\
\hline E-BP & 22.18 & 10.3 \\
\hline $\mathrm{M}-\mathrm{CF}$ & 0.9 & 133 \\
\hline M-D & 0.7 & 13.6 \\
\hline M-SH & 4.5 & 47.7 \\
\hline $\mathrm{M}-\mathrm{VE}$ & 0.0 & 103.7 \\
\hline M-SA & 146.4 & 56.3 \\
\hline M-BP & 0.3 & 14.3 \\
\hline CF-D & 1.7 & 104.8 \\
\hline CF-SH & 4.2 & 44.2 \\
\hline CF-VE & 0.5 & 0.7 \\
\hline CF-SA & 13.6 & 48.56 \\
\hline CF-BP & 0.1 & 37.3 \\
\hline D-SH & 0.3 & 17.7 \\
\hline D-VE & 0.3 & 71.7 \\
\hline D-SA & 42.5 & 24.5 \\
\hline D-BP & 1.1 & 1.2 \\
\hline SH -VE & 1.3 & 26.2 \\
\hline SH-SA & 96.9 & 0.0 \\
\hline SH-BP & 3.3 & 2.7 \\
\hline VE-SA & 22.1 & 27.5 \\
\hline VE-BP & 0.15 & 26.7 \\
\hline SA-BP & 25.7 & 3.7 \\
\hline
\end{tabular}

clusters of different terpenic categories, while the $\mathrm{FW}$ of both PC1 and PC2 showed still significant but lower values within classes of resins belonging to the same category, except for colophony and Venice turpentine. Moreover, it was extremely interesting to find out that the low FW values $(\mathrm{PC} 1,1.1 ; \mathrm{PC} 2,1.2)$ between the $\mathrm{BP}$ sample and the dammar spectra confirm the characterization of the unknown varnish as dammar. 


\section{Conclusion}

This paper allowed to propose FarIR-ATR spectroscopy as a complementary tool for the identification of natural resins. Compared with MidIR spectroscopy, FarIR analyses allow to better distinguish sesquiterpenic, diterpenic, diterpenic with polymerised communic acid and triterpenic resins. Moreover, PCA of spectral data permits sometimes (i.e. dammar and mastic, shellac and elemi) to distinguish between resins belonging to the same category, although an evaluation of the spectral changes in resin derived by the oxidation and hydrolysis process occurring with ageing is being carried out to better understand the technique diagnostic performances.

Even though ageing effect has not been evaluated yet, the method has been tested on historical case studies. In the first case (sample BP) this method allowed to confirm the results obtained with analytical pyrolysis. On sample PMB7, the bulk analyses approach was not effective in the identification of the resin due to the strong signals arising from the leather support. In this case, FarIR spectroscopy allowed the identification of the resin. Actually, thanks to its flexibility PMB7 sample did not disaggregate after the contact with the crystal and the analyses could be specifically focused on the external surface, even though a small influence of the leather support is present.

FIR spectroscopy has the disadvantage of producing less intense spectra and of being difficult to perform on a microscopic scale. It can be therefore used as a complementary technique of Raman spectroscopy and of the traditional FTIR spectroscopy in the MidIR region.

A particularly interesting field of application may be the control of the cleaning procedures during restoration.

Acknowledgements Part of this research has been funded by the national project PRIN 08 "Setting up of diagnostic methodologies for the stratigraphical characterisation and spatial location of the organic components in artistic and archaeological polychrome works of art" and by the European project "CHARISMA" Cultural heritage Advanced Research Infrastructures: Synergy for a Multidisciplinary Approach to Conservation/Restoration, FP7 INFRASTRUCTURE n.228330.

\section{References}

1. Thompson DV Jr (1954) Il libro dell'arte, the craftsman's handbook of Cennino d'Andrea Cennini. Dover, New York

2. Thomson R. (2005) In Conservation of leather and related materials, Buttherworth Heinemann, 88-91

3. Mills J, White R (1999) Organic material in museum objects, 2dth edn. Butterworth Heinemann, Oxford, pp 95-129

4. Andreotti A, Bonaduce I, Colombini MP, Gautier G, Modugno F, Ribechini E (2006) Anal Chem 78:4490-4500

5. Domènech-Carbò MT, Kuckova S, de la Cruz-Canizares J, OseteCortina L (2006) J Chrom A 1121:248-258

6. Doménech-Carbò MT (2008) Anal Chim Acta 621:109-139

7. van den Berg JDJ, Boon JJ, van den Berg KJ, Fiedler I, Miller MA (1998) Anal Chem 70:1823-1830
8. Chiavari G, Fabbri D, Prati S (2002) Chromatographia 55:611-616

9. Shedrinsky SM, Wampler TP, Baer NS (1987) Wiene Berichte veber Naturwissenschaft in der Kunst 4:12-25

10. Osete-Cortina L, Domènech-Carbò MT (2005) J Chromatogr A 1065:265-278

11. Manso M, Carvalho ML (2009) Spectr Acta B 64:482

12. Casadio F, Toniolo L (2001) J Cult Heritag 2:71-78

13. Messerschmidt RG, Harthcock MA (1988) Infrared microspectroscopy. Theory and applications. Marcel Dekker, New York

14. Ricci C, Miliani C, Brunetti BG, Sgamellotti A (2006) Talanta 69:1221-1226

15. Bacci M (1995) Sens Actuat B 29(1-3):190-196

16. Katsibiri O, Howe RF (2010) Microchem J 14-23

17. Feller RL (1954) Science 120:1069-1070

18. Low MJD, Baer NS (1978) In: Conference Proceedings ICOM Committee for Conservation. 5th Triennial Meeting, Zagreb, 1-8 Oct. 1978., Paris 1978

19. Cartoni G, Russo MV, Spinelli F, Talarico F (2003) Annali di Chimica 93:849-861

20. Scalarone D, Lazzari M, Chiantore O (2003) J Anal Appl Pyrol 68-69:115-136

21. Nevin A, Comelli D, Osticioli I, Toniolo T, Valentini G, Cubeddu R (2009) Anal Bioanal Chem 395:2139-2149

22. Edwards HGM, David AR, Brody RH (2008) J Raman Spectr 39:966-971

23. Edwards HGM, Falk MJ, Quye A (1997) J Raman Spectrosc 28:243-249

24. Edwards HGM, Sibley MG, Heron C (1997) Spectrochim Acta Part A 53:2373-2382

25. Edwards HGM, Farwel DW, Daffner PL (1996) Spectrochim Acta A $52: 1639-1648$

26. Brody RH, Edwards HGM, Pollard AM (2002) Biospectr 67:129-141

27. Brody RH, Edwards HGM, Pollard AM (2001) Spectrochim Acta A $57: 1325-1338$

28. Vandenabeele P, Ortega-Aviles M, Castilleros DT, Moens L (2007) Spectrochim Acta A 68:1085-1088

29. Vandenabeele P, Grimaldi DM, Edwards HGM, Moens L (2003) Spectrochim Acta A 59:2221-2229

30. Vandenabeele P, Wehling B, Monees L, Edwards E, de Rev M, van Hooydonk G (2000) Anal Chim Acta 407:261-274

31. Lau D, Livett M, Prawer S (2008) J Raman Spectr 39:545-552

32. Karr C, Kovach JJ (1969) Appl Spectrosc 23:219-223

33. Kendix E, Moscardi G, Mazzeo R, Baraldi P, Prati S, Joseph E, Capelli (2008) J Raman Spectrosc 39:1104-1112

34. Kendix EL, Prati S, Joseph E, Sciutto G, Mazzeo R (2009) Anal Bioanal Chem 394:1023-1032

35. Vahur S, Knuutinen U, Leito I (2009) Spectrochim Acta A 73:764-771

36. Vahur S, Knuutinen U, Leito I (2010) Spectrochim Acta A 75:1061-1072

37. Prati S, Joseph E, Sciutto G, Mazzeo R (2010) Acc Chem Res 43:792-801

38. Barnes J, Dhanoa MS, Lister SJ (1989) App Spectrosc 43:772-777

39. Torri C, Fabbri D (2009) Microchem J 93:133-139

40. Mazzeo R, Joseph E, Prati S, Millemaggi A (2007) Anal Chim Acta 599:107-117

41. Dutta S, Mallick M, Bertram N, Greenwood PF, Mathews RP (2009) Intern J Coal Geol 80:44-50

42. Esteban M, Arino C, Diaz-Cruz JM (2006) Trends Anal Chem 25:86-92

43. Harper AM, Duewer DL, Kowalski BR, Fashing JL (1977) In Kowalski BR (ed) Chemometrics: Theory and Application, ACS Symposium

44. Oliveri P, Baldo MA, Daniele S, Forina M (2009) Anal Bioanal Chem 395:1135-1143 